\title{
Examination of Patterns in Intubation by an Emergency Airway Team at a Large Academic Center: Higher Frequency During Daytime Hours
}

\author{
M Christopher Adams MD, Ulrich Schmidt MD PhD, Dean R Hess PhD RRT FAARC, \\ Henry T Stelfox MD PhD, and Edward A Bittner MD PhD
}

\begin{abstract}
BACKGROUND: Emergency airway management represents an event with high acuity but unpredictable frequency and therefore presents a challenge for adequate staffing. Given circadian and seasonal variations, we hypothesized that the majority of emergency airway events happen after normal working hours and during the winter months. METHODS: A retrospective analysis of 1,482 intubations by an emergency airway team over a 3-y period was performed. The data were obtained from hospitalized patients who required emergency airway management in a large academic medical center. A database of emergency airway consultations was analyzed for intubation time and date information, as well as geographic location within the hospital. RESULTS: A greater percentage of emergency intubations occurred during day shift hours (7 AM to $7 \mathrm{PM}$ ) compared with night shift hours, $57 \%$ and $43 \%$, respectively $(P<.01)$. The monthly frequency of intubations was not uniformly distributed across the year $(P<.01)$. The greatest percentage of intubations was performed in February (10.9\%), with the lowest being recorded in August (4.7\%). CONCLUSIONS: Emergency airway service utilization is highest during daytime hours, with seasonal variations composed of higher consults in the winter and lower consults in the summer. Key words: intubation; airway management; resuscitation; personnel staffing and scheduling. [Respir Care 2014;59(5):743-748. (C) 2014 Daedalus Enterprises]
\end{abstract}

\section{Introduction}

The immediate availability of an in-hospital airway consult service is a cornerstone of providing safe care for patients who require emergency airway management. Need for emergency airway support is a critical patient event,

Drs Adams, Schmidt, and Bittner are affiliated with the Department of Anesthesia, Critical Care and Pain Medicine, and Dr Hess is affiliated with the Department of Respiratory Care, Massachusetts General Hospital, Boston, Massachusetts. Dr Stelfox is affiliated the Departments of Critical Care Medicine, Medicine, and Community Health Sciences, Institute for Public Health, University of Calgary, Alberta, Canada.

Dr Hess discloses relationships with Philips Respironics, Pari, Covidien, Maquet, Merck, and Bayer. The other authors have declared no conflicts of interest.

Dr Adams presented an abstract of this study at the Society of Critical Care Medicine Annual Congress, held January 15-19, 2011, in San Diego, California. with potentially devastating consequences if the need is not addressed immediately. High acuity patient events with unpredictable frequency, such as emergency airway management, present challenges for allocating staffing and other resources.

Many investigators have studied the optimization of resource utilization in the setting of uncommon or unpredictable events. Examples include more common scenarios, such as predicting trauma admissions based on seasonal and weather data, ${ }^{1}$ to more hypothetical events, such as assessing readiness to manage mass casualties following attack with weapons of mass destruction. ${ }^{2}$ Prediction of staffing patterns remains important, as staffing strategies

\footnotetext{
Correspondence: M Christopher Adams MD, Department of Anesthesia, Critical Care and Pain Medicine, Massachusetts General Hospital, 55 Fruit Street, Boston, Massachusetts 02114. E-mail: adamsmc@ gmail.com.
}

DOI: $10.4187 /$ respcare. 02432 


\section{Patterns in Intubation by an Emergency Airway Team at a Large Academic Center}

have been shown to have an impact on patient morbidity and mortality. ${ }^{3}$ With a particular focus on the management of emergency airway support, researchers have shown that adequate staffing exerts a limiting effect on the adverse impact of emergency airway events. ${ }^{4,5}$ However, there are no papers reporting the frequencies of such events; clarifying this distribution may help administrators match resources to needs.

Circadian variation in the frequency of medical emergencies has been reported. ${ }^{6-9}$ We therefore hypothesized that the majority of airway emergencies would follow this pattern, with the greatest number of airway consults occurring before the start of a standard work day, particularly during late night and early morning hours. Given the usual reduction in resources during such hours, an increase in emergency airway events would have potential implications for staffing adequacy.

Additionally, we examined seasonal variation in airway consults. Since the frequency of respiratory diseases increases during the winter months, ${ }^{10,11}$ we hypothesized that there would be a higher need for emergency airway management during this time period.

To test our hypotheses, we utilized a database of 1,482 intubations performed over a 3-y period at a large academic medical center. The data were analyzed for temporal differences in frequency of emergency intubations.

\section{Methods}

From September 2007 through June 2010, data from emergency and urgent intubations were collected at a large academic medical center, the Massachusetts General Hospital (MGH), in Boston, Massachusetts. The study was approved by the hospital's Institutional Review Board.

MGH is a 900-bed university-affiliated teaching hospital. It serves as a tertiary and quaternary referral center, a Level 1 trauma center, and a community hospital for the city of Boston and nearby suburbs. Each year, there are $\sim 1.5$ million patient visits and 45,000 admissions. During the data collection period, there were 8 adult ICUs, which could accommodate a total of 130 patients.

At MGH, the "emergency airway team" is a consultative service based in the surgical ICU (SICU). It is composed of an anesthesia resident rotating in the SICU, a critical care fellow, a staff intensivist, and a respiratory therapist.

Emergency airway consultations may result in intubation or in recommendations to the primary team to help otherwise optimize a patient with respiratory compromise. Only airway consultations that resulted in endotracheal intubation were included in these analyses. For the purposes of discussion, these events will be referred to as "intubations."

\section{QUICK LOOK}

\section{Current knowledge}

The need for emergency airway support is a critical patient event, with potentially devastating consequences if the need is not addressed immediately. An in-house service to provide this expertise is important for patient safety.

\section{What this paper contributes to our knowledge}

Emergency intubation is a common but unpredictable event. The frequency of emergency intubations is highest during day shift hours. There was a seasonal fluctuation, with more consults in the winter and early spring months and fewer consults in the summer months.

In the emergency ward, the emergency medicine staff is primarily responsible for airway management; such intubations were not captured in our analysis. However, the emergency airway team is occasionally brought to the emergency ward to perform intubations; these particular events were included in the data set.

With the exceptions listed above, the emergency airway team is responsible for management of all emergency airway consultations in the adult hospital. Intubations performed in the SICU, where the emergency airway team is based, were included in the data.

Data were analyzed for variations in frequency across several time divisions. The first analysis involved dividing a 24-h day into day and night shift periods, with the day shift representing the hours of 7 AM to 7 PM. The second analysis involved dividing a 24-h day into six 4-h tranches to assess variations in consult frequency throughout the day. These tranches included 3-7 AM, 7-11 AM, 11 AM to 3 PM, 3-7 PM, 7-11 PM, and 11 PM to 3 AM. Tranches were inclusive of the start time and exclusive of the end time. In a third analysis, month-to-month variations were studied. For this purpose, data were normalized for months of available data.

To determine the influence of the number of patients in the hospital, we analyzed monthly variations in intubation frequency after normalizing the intubation data set against the monthly hospital census recorded during the data collection period.

In addition to temporal information, data were available regarding the indication for intubation. The categories available to describe the indication included respiratory compromise, hemodynamic compromise, trauma/airway protection, and other. Categorical selection was not mutually exclusive.

Finally, the method of endotracheal intubation (eg, Miller blade, fiberoptic bronchoscope) and Cormack-Lehane la- 


\section{Patterns in Intubation by an Emergency Airway Team at a Large Academic Center}

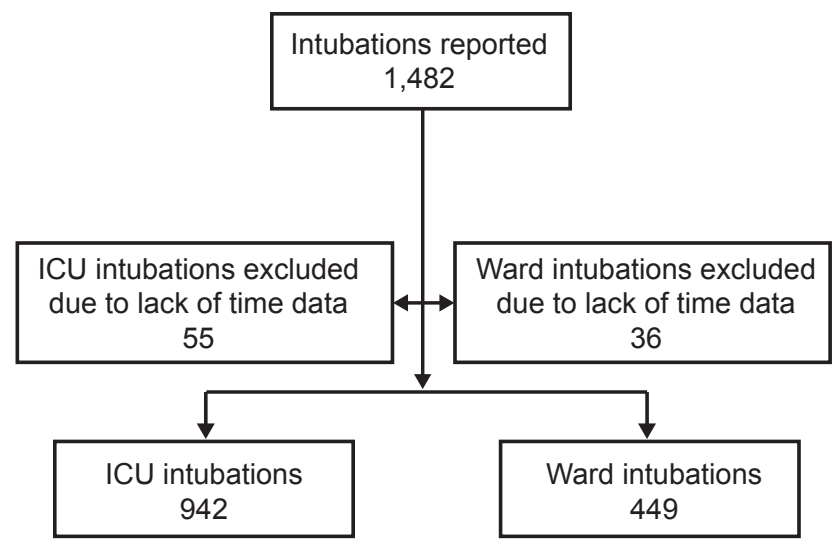

Fig. 1. Dataset composition flowchart. Data are shown for intubations included in the hourly analysis only. Flowchart does not display distribution of intubations included in monthly analysis.

ryngoscopic classification were recorded. As with indication, these categorizations were not mutually exclusive.

\section{Statistical Analysis}

Data were compiled (Excel, Microsoft, Redmond, Washington) and analyzed (Stata, StataCorp, College Station, Texas). Chi-square analyses were performed to assess variations across temporal categories. The null hypothesis was that the frequency of intubations was uniformly distributed across the time category of interest. $P<.05$ was considered to be significant.

\section{Results}

The total number of intubations recorded was 1,482, representing $\sim 1.5$ intubations per day over the course of the study period. Data entries included date and time of the intubation, as well as location within the hospital. Location data differentiated between ICU (1,003 intubations) and ward (415 intubations) patients, with 64 intubations lacking complete location information. Data points that lacked information regarding date (9 intubations) and/or time (91 intubations) were excluded from the analysis (Fig. 1).

Time data were available for 1,391 intubations (94\% of the total). Of these, 799 (57\%) occurred during day shift hours (7 AM to $7 \mathrm{PM}$ ), and 592 (43\%) occurred during night shift hours $(P<.01)$. Comparing airway consult frequency across a divided day, most intubations occurred between 3 and $7 \mathrm{PM}(n=286,21 \%$ of total). The least number of intubations occurred between $11 \mathrm{PM}$ and $3 \mathrm{AM}(n=161$, $12 \%$ of total). The frequency of intubations performed across the six 4-h tranches was not uniformly distributed $(P<.01)$. Intubation frequency was homogeneously distributed across the three 4-h tranches composing the day shift $(P=.06)$. However, the frequency was not homogeneously distributed across the three tranches composing the night shift $(P<.01)$. Figure 2 displays the frequency of intubations over the course of a 24-h day.

We further analyzed monthly variations of emergency airway management. Date information was available for 1,473 intubations. The highest monthly average of intubations was recorded for February $(n=57,10.9 \%$ of the average yearly total), with the fewest being in August ( $n=25,4.7 \%$ of the average yearly total). The monthly frequency of intubations was not uniformly distributed across the year $(P<.01)$.

Finally, we analyzed monthly intubation frequency after normalizing the data for the monthly hospital census. Census data showed that the highest average number of hospital admissions occurred in the month of April $(4,859)$, with the lowest occurring in February $(4,330)$ and September $(4,545)$. Figure 3 displays the frequency of intubations as distributed over the course of a 12-mo year, normalized against admissions.

Of the intubations recorded, the indications for intubation were specified for the following categories: respiratory compromise (1,014 intubations), hemodynamic compromise (62 intubations), trauma/airway protection (226 intubations), and other (158 intubations). Included in the previous totals were 172 intubations for which multiple indications were specified. For 218 intubations, no indication was listed.

The method of endotracheal intubation was also available for most intubations. This included direct laryngoscopy using a MacIntosh blade (1,209 intubations) or Miller blade (93 intubations) and fiberoptic bronchoscopy ( 25 intubations). For 12 of the previously listed intubations, multiple modalities were specified. For 169 intubations, the method was not specified.

When direct laryngoscopy was performed, the CormackLehane laryngoscopic classification was listed. These classifications were Grade I (full view of glottis, 994 intubations), Grade II (partial view of glottis, 219 intubations), Grade III (view only of epiglottis, 98 intubations), and Grade IV (no view of airway structures, 24 intubations). For 4 of the previously listed intubations, multiple Cormack-Lehane views were recorded. For 131 intubations, no view was recorded.

Intubations with a laryngoscopic grade of III and/or IV, as well as intubations requiring a fiberoptic bronchoscope, were assumed to be difficult. Taken as independent intubations, the total of probable difficult airways was 142, a $10.5 \%$ incidence of difficult airway. Timestamps were available for 131 of the 142 intubations with presumed difficult airways (92\%). A nonsignificant majority of these difficult airways were encountered during the day shift hours of 7 AM to $7 \mathrm{PM}(n=75,57.3 \%)$, with the remainder occurring during night shift hours $(n=56,42.7 \%, P=.10)$. 


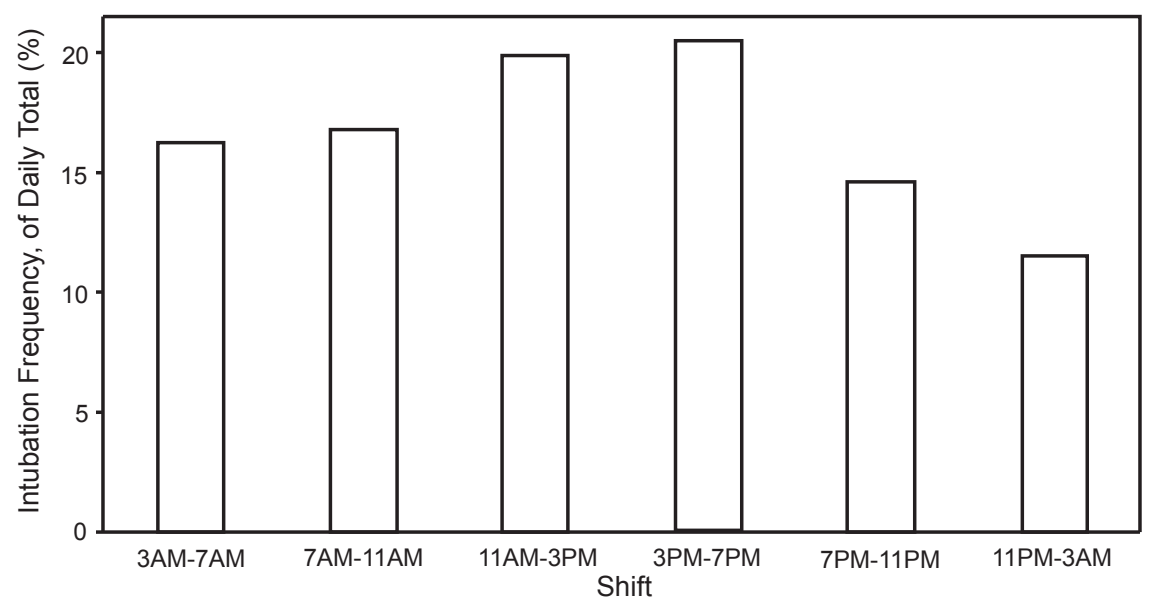

Fig. 2. Intubation frequency by shift. A percentage of the daily total intubations by the emergency airway team is displayed for each 4-hour tranche.

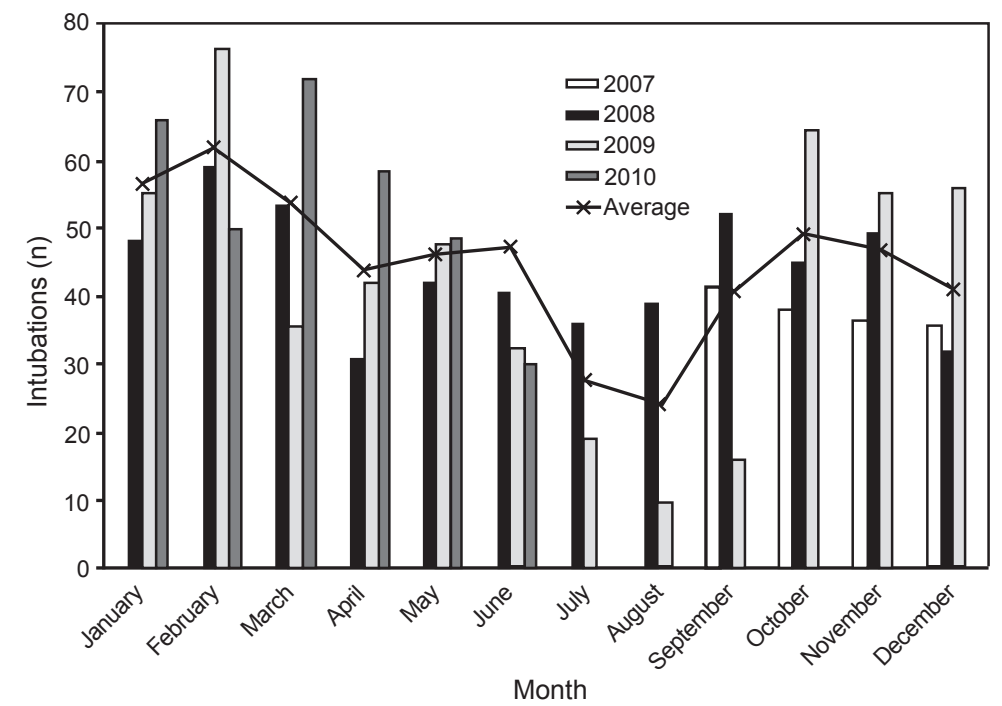

Fig. 3. Monthly intubation frequency, normalized for hospital census. The number of intubations by the emergency airway team is displayed for each month and year, as a total per 4,700 admissions (the average monthly admissions during the study period). The trendline represents the monthly average intubations for all available data.

These intubations were uniformly distributed throughout the course of the day $(P=.29)$ (data represented in Fig. 4).

\section{Discussion}

The principal finding of our study is that the majority of urgent and emergency intubations take place during the daytime hours of 7 AM to 7 PM. Furthermore, a majority of airway consultations for intubation occur during the winter and early spring months of January through March.

Although the majority of intubations by emergency airway services take place during daytime hours, it is important to note that a clinically important $43 \%$ of intubations take place during night shift hours. This fact stands in contrast to analyses of call patterns to medical emergency teams (rapid response teams); some studies have shown a higher frequency of calls during night shift hours. ${ }^{12}$

When looking at a 24-h day with finer resolution, we have shown that the highest frequency of intubations occurs during the hours of 3 PM to 7 PM, with the lowest frequency occurring during the hours of 11 PM to $3 \mathrm{AM}$. It is impossible to determine from these data the explanation for such a drop in frequency overnight, although there is evidence to support circadian variations in the frequency of medical emergencies. ${ }^{6}$ An alternative possibility would be diurnal variations in the extent of attention and therapeutic interventions delivered to patients, perhaps as a calculated tradeoff to improve sleep quality and quan- 


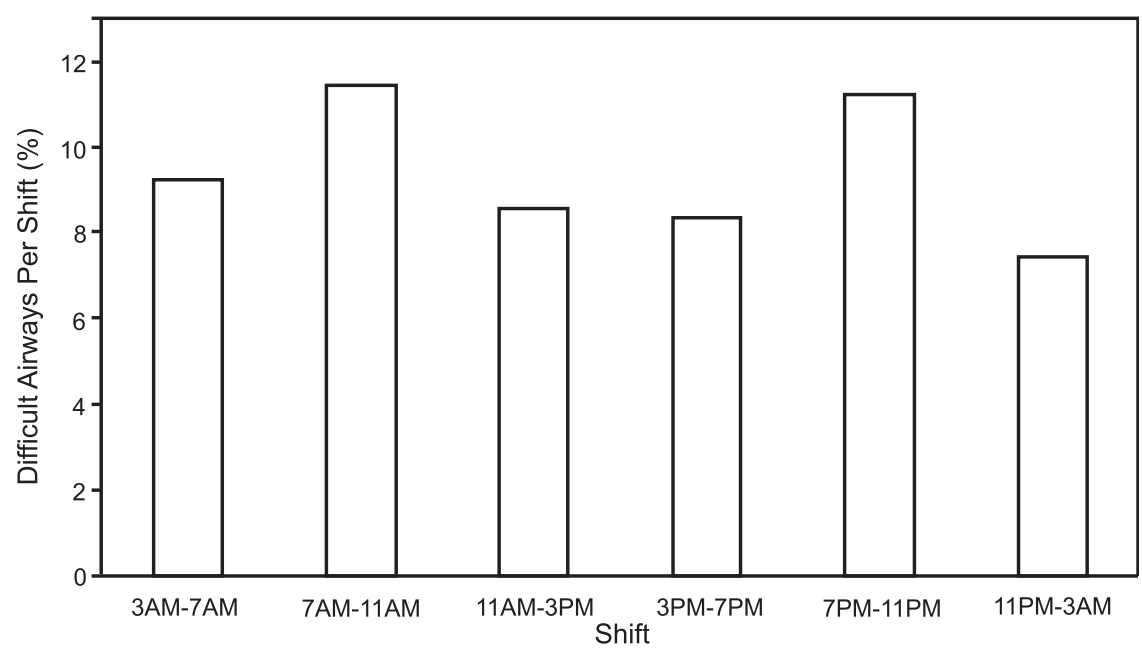

Fig. 4. Difficult airway frequency by 4-hour tranche. Presumed difficult intubations are displayed as a percentage of each tranche's total intubations. Cormack-Lehane classifications III and IV and use of fiberoptic bronchoscopy were used as proxies for difficulty.

tity. ${ }^{13,14}$ This phenomenon has been witnessed when studying calls to medical emergency teams ${ }^{15}$ and might imply that intubations that should have been performed overnight are instead delayed until daybreak. Additional study is warranted to determine whether a significant number of early-morning airway consults are performed for patients with more severe respiratory distress or whether this cohort of patients suffer worse clinical outcomes.

We observed a drop in intubations during the summer months of July through September, with an increase during the winter and spring months of January through May. This may relate to the increased incidence of respiratory illness witnessed during the winter and early spring months. It also correlates with an elevated hospital census during these months, although this increase alone does not account for the higher number of airway consults (see Fig. 3).

Additionally, the highest monthly frequency of emergency airway consults does not coincide with the introduction of new trainees, mitigating the consequences of any such so-called "July Effect" 16 on airway events.

Some narrow conclusions may be drawn about the incidence and frequency of difficult intubation encountered at the study institution. When using proxy indicators for airway difficulty, we showed a nearly $11 \%$ incidence, on the lower end of the reported range of $11-25 \%$ for outof-operating room difficult intubations. ${ }^{17-19}$ Our data displayed an even proportional distribution of these presumed difficult intubations across a 24-h day; it is reassuring that this frequency did not increase during off-shift hours, when resources are fewer.

Limitations to the study include the fact that this is a retrospective analysis. It is unknown whether similar patterns exist at other hospitals of different types (eg, nonacademic) and sizes. Additionally, the composition of the emergency airway team and its responsibilities may be significantly different at other institutions.

The database does not include outcome data, and therefore, the study cannot address whether there is a seasonal or hourly variation in complications or difference in outcomes of emergency airway management. The database does not include airway consultations that did not result in intubation, so resource utilization is not captured for "nonurgent" airway consultations, which result in therapies such as noninvasive positive-pressure ventilation.

Overall, these data show moderate variation in the frequency of emergency airway consults in the hospital: a relatively common event at 1.5 intubations per day at the 900-bed study institution. Our analysis shows that demand for emergency airway support drops during the evening hours, a fact that may indicate that nighttime airway consult resources do not need to be augmented above daytime levels. This recommendation would be tempered, however, if additional studies showed that a drop in overnight emergency airway consult frequency is explained by the delay of intubation that was clinically indicated earlier.

\section{Conclusions}

Need for emergency intubation is a common but unpredictable event. The frequency of intubations by emergency airway consult services is highest during day shift hours. There is a seasonal fluctuation composed of higher consults in the winter and early spring months of January through March, with decreased frequency in the summer months of June through August.

\section{REFERENCES}

1. Rising WR, O'Daniel JA, Roberts CS. Correlating weather and trauma admissions at a level I trauma center. J Trauma 2006;60(5):10961100 . 


\section{Patterns in Intubation by an Emergency Airway Team at a Large Academic Center}

2. Candiotti KA, Kamat A, Barach P, Nhuch F, Lubarsky D, Birnbach DJ. Emergency preparedness for biological and chemical incidents: a survey of anesthesiology residency programs in the United States. Anesth Analg 2005;101(4):1135-1140.

3. Pronovost PJ, Angus DC, Dorman T, Robinson KA, Dremsizov TT, Young TL. Physician staffing patterns and clinical outcomes in critically ill patients: a systematic review. JAMA 2002;288(17):2151-2162.

4. Schmidt UH, Kumwilaisak K, Bittner E, George E, Hess D. Effects of supervision by attending anesthesiologists on complications of emergency tracheal intubation. Anesthesiology 2008;109(6):973-977.

5. Needham DM, Thompson DA, Holzmueller CG, Dorman T, Lubomski $\mathrm{LH}, \mathrm{Wu} \mathrm{AW}$, et al. A system factors analysis of airway events from the Intensive Care Unit Safety Reporting System (ICUSRS). Crit Care Med 2004;32(11):2227-2233.

6. Arntz HR, Willich SN, Oeff M, Brüggemann T, Stern R, Heinzmann A, et al. Circadian variation of sudden cardiac death reflects agerelated variability in ventricular fibrillation. Circulation 1993;88(5 Pt 1):2284-2289.

7. Brenner BE, Chavda KK, Karakurum MB, Karras DJ, Camargo CA Jr, MARC Investigators. Circadian differences among 4,096 emergency department patients with acute asthma. Crit Care Med 2001; 29(6):1124-1129.

8. Muller JE. Circadian variation and triggering of acute coronary events. Am Heart J 1999;137(4 Pt 2):S1-S8.

9. Tsai CL, Brenner BE, Camargo CA Jr. Circadian-rhythm differences among emergency department patients with chronic obstructive pulmonary disease exacerbation. Chronobiol Int 2007;24(4):699-713.

10. Fry AM, Curns AT, Harbour K, Hutwagner L, Holman RC, Anderson LJ. Seasonal trends of human parainfluenza viral infections: United States, 1990-2004. Clin Infect Dis 2006;43(8):1016-1022.
11. Simonsen L, Reichert TA, Viboud C, Blackwelder WC, Taylor RJ, Miller MA. Impact of influenza vaccination on seasonal mortality in the US elderly population. Arch Intern Med 2005;165(3):265-272.

12. Jones D, Bates S, Warrillow S, Opdam H, Goldsmith D, Gutteridge G, Bellomo R. Circadian pattern of activation of the medical emergency team in a teaching hospital. Crit Care 2005;9(4):R303-R306.

13. Weinhouse GL, Schwab RJ, Watson PL, Patil N, Vaccaro B, Pandharipande $\mathrm{P}$, et al. Bench-to-bedside review: delirium in ICU patients-importance of sleep deprivation. Crit Care 2009;13(6):234.

14. Inouye SK, Bogardus ST Jr, Charpentier PA, Leo-Summers L, Acampora D, Holford TR, Cooney LM Jr. A multicomponent intervention to prevent delirium in hospitalized older patients. N Engl J Med 1999;340(9):669-676.

15. Galhotra S, DeVita MA, Simmons RL, Schmid A. Impact of patient monitoring on the diurnal pattern of medical emergency team activation. Crit Care Med 2006;34(6):1700-1706.

16. Phillips DP, Barker GE. A July spike in fatal medication errors: a possible effect of new medical residents. J Gen Intern Med 2010; 25(8):774-779.

17. De Jong A, Molinari N, Terzi N, Mongardon N, Arnal JM, Guitton $\mathrm{C}$, et al. Early identification of patients at risk for difficult intubation in the intensive care unit: development and validation of the MACOCHA score in a multicenter cohort study. Am J Respir Crit Care Med 2013;187(8):832-839.

18. Heuer JF, Barwing TA, Barwing J, Russo SG, Bleckmann E, Quintel $\mathrm{M}$, et al. Incidence of difficult intubation in intensive care patients: analysis of contributing factors. Anaesth Intensive Care 2012;40(1): 120-127.

19. Griesdale DE, Chau A, Isac G, Ayas N, Foster D, Irwin C, et al. Video-laryngoscopy versus direct laryngoscopy in critically ill patients: a pilot randomized trial. Can J Anaesth 2012;59(11):1032-1039. 